\title{
perifèria
}

Número 19 (2), Diciembre 2014

revistes.uab.cat/periferia

\section{Narrativas de la movilidad cotidiana: un caso de estudio en el barrio La Maternitat i San Ramon de \\ Barcelona}

\author{
Alejandro Román Mosquera - Universidad Autónoma de Barcelona ${ }^{1}$
}

DOI: http://dx.doi.org/10.5565/rev/periferia. 441

\begin{abstract}
Resumen
La movilidad urbana cotidiana es una experiencia vital a nivel individual y colectivo, es el resultado de una realidad compleja que involucra variables territoriales y socioeconómicas. El nuevo paradigma de la movilidad surge con el siglo XXI desde las Ciencias Sociales tanto desde la perspectiva del análisis de la distancia y el tiempo, como desde la experiencia individual, cargada de significados. Este artículo que resume un estudio de caso, plantea cuáles son las narrativas culturales que están asociadas con la movilidad cotidiana y cómo estás podrían tener un grado de relación con el reparto modal y con la percepción de la movilidad urbana en Barcelona.
\end{abstract}

Palabras clave: Movilidad urbana, reparto modal, cultura urbana, Barcelona

\begin{abstract}
Daily urban mobility is a vital experience at an individual as well as collective level and is the result of a complex reality that involves both territorial and socioeconomic variables. "The Mobility turn" emerged in the 21st Century from within the Social Sciences as a way of analysing distance and time as well as an individual's meaningful experience. This article summarizes a case study that explains what cultural narratives associate with urban mobility and how these narratives could have a connection with the modal choice and the perception of daily urban mobility in Barcelona.
\end{abstract}

Keywords: Urban mobility, mode choice, urban culture, Barcelona

\footnotetext{
${ }^{1}$ Enviar correspondencia a: Alejandro Román. alejandro.roman2@gmail.com
} 


\section{perifèria}

Número 19 (2), Diciembre 2014

revistes.uab.cat/periferia

\section{Introducción}

El tiempo es uno de los valores de la vida moderna y su gestión depende en gran medida de la movilidad: de los desplazamientos cotidianos que se realizan para acceder a un bien, a un servicio o para la realización de actividades (MirallesGuasch \& Freixa, 2008).

La movilidad cotidiana en tanto que fenómeno urbano es una experiencia vital, tanto a nivel individual como colectivo. Esta experiencia es el resultado de una realidad compleja que involucra variables territoriales y socioeconómicas (MirallesGuasch \& Cebollada, 2009) y en las que la condición individual del viajero y su relación con el espacio de tránsito son relevantes. De hecho, esta realidad se constituye como un nuevo paradigma de estudio para diversas áreas del conocimiento, entre ellas la antropología (Sheller \& Urry, 2006).

Así, el nuevo paradigma de la movilidad surge con el siglo XXI desde las Ciencias Sociales tanto desde la perspectiva del análisis de la distancia y el tiempo, como desde la experiencia individual, cargada de significados e inmersa en una red de relaciones. En este campo, las nuevas tecnologías de la información juegan un papel preponderante, al relativizar las distancias y coadyuvar a la creación y recreación de narrativas alrededor de los desplazamientos.

\section{Distancia y tiempo}

Distancia y tiempo como vectores de la velocidad han sido los ejes del desarrollo de la movilidad en las últimas tres décadas, marcadas por la masificación del automóvil. Este invento revolucionario es omnipresente cuando se piensa en términos de escala del territorio (Featherstone, Thrift, \& Urry, 2005; Hagman, 2003; Jacobs, 2013; Kaufmann, 2002; Kenworthy \& Laube, 1999; Sheller \& Urry, 2000).

En este sentido, el automóvil, considerado el motor de la modernidad y el símbolo del progreso de la sociedad capitalista, ha sido la inspiración para el diseño y distribución de las funciones de movilidad de las ciudades. No es una novedad reconocer que en la gran mayoría de regiones metropolitanas del mundo el impacto 


\section{perifèria}

Número 19 (2), Diciembre 2014

revistes.uab.cat/periferia

del automóvil es absoluto, con todo lo que ello implica en cuanto a la dependencia de combustibles fósiles, especialmente en territorios caracterizados por su baja densidad (Kenworthy \& Laube, 1999), en problemas medioambientales y pérdidas socioeconómicas relacionadas con las retenciones de tráfico.

En este contexto, las personas que viajan toman sus decisiones en un espacio de disponibilidad de alternativas de transporte y de significación de éstas. Estos desplazamientos no deben analizarse únicamente con un enfoque instrumental relacionado con el uso de un medio de transporte y las distancias recorridas sino como decíamos, incorporando las motivaciones de los viajeros.

La movilidad de acuerdo con Cresswell (2006), clasifica el cómo, el cuándo y por dónde se mueven los sujetos, en la misma jerarquía en la que se ubica el lugar de residencia, debido a que los espacios de tránsito confluyen fuerzas internas y externas e imaginarios culturales que configuran la experiencia del viaje. Estas jerarquías están relacionadas con categorías sociales, económicas y culturales que van a determinar quiénes tienen mayores posibilidades de moverse con fluidez y de manera confortable.

Con el objeto de explorar esta cuestión, presentamos un estudio de caso desde la perspectiva antropológica en el barrio La Maternitat i San Ramon, de Barcelona, cuyo objetivo es analizar las dimensiones culturales asociadas a las narrativas de la movilidad cotidiana y cómo éstas pueden influir en la gradación del reparto modal (o uso de diferentes tipos de transporte) de la ciudad.

Para comenzar abordaremos la literatura sobre el nuevo paradigma de la movilidad. Seguidamente presentaremos las particularidades de la movilidad en Barcelona, en base a los datos de la Encuesta de Movilidad en Días Laborables (EMEF2012) (IERMB, 2012). A continuación, presentaremos la metodología que describe cómo fue planeado el trabajo de campo, las características generales, los procedimientos y materiales relacionados. Una vez presentada la metodología expondremos los resultados y el análisis de los datos con el fin de lograr una aproximación a las narrativas culturales 


\section{perifèria}

Número 19 (2), Diciembre 2014

revistes.uab.cat/periferia

asociadas a la movilidad cotidiana en Barcelona. Por último, presentaremos una serie de conclusiones sobre algunos de los conceptos que permiten establecer cuáles son las narrativas asociadas a la movilidad cotidiana en una zona geográfica de Barcelona.

\section{EI nuevo paradigma de la movilidad}

Tal como explica Sennett (1997), el sistema de transporte ha tenido por objetivo mejorar las conexiones en el interior de las ciudades y su periferia, creando un espacio intenso de movilidad. En general, en la geografía del transporte se estudian una serie características socioeconómicas (Passalacqua, A. Batteau, 2014) y territoriales (Gutiérrez \& García-Palomares, 2007: 18; Banister, 2011: 1541) que definen patrones de movilidad, como son la opción modal (modal choice) y el comportamiento de los viajeros para realizar sus desplazamientos cotidianos (Klinger, Kenworthy, \& Lanzendorf, 2013:19), lo que acaba configurando el reparto modal (modal split) de una ciudad o región.

Las variables económicas, según explica Miralles-Guasch \& Cebollada (2009), se refieren a los ingresos monetarios, la edad, el sexo, el tiempo, la historia y otros factores culturales que puedan influir en la selección de un medio de transporte sobre otro. Los ingresos del sujeto determinan directamente la opción modal, de acuerdo con Passalacqua \& Batteau (2014).

Las variables territoriales se refieren a la estructura del territorio, la densidad urbana, la infraestructura de transporte, la distribución espacial, la multifuncionalidad y el ordenamiento del territorio.

La movilidad como práctica social, por último, es el resultado de estas variables socioeconómicas y territoriales combinadas con la intensidad/capacidad de los individuos para movilizarse.

\section{La movilidad: una experiencia compleja}

Muchas de las interacciones sociales son relaciones de proximidad y lejanía que motivan o necesitan desplazamientos para ser mantenidas. De acuerdo con Sheller 


\section{perifèria}

Número 19 (2), Diciembre 2014

revistes.uab.cat/periferia

\& Urry (2006), con la cosmopolitización de una parte del mundo se crean nuevas necesidades que motivan nuevos viajes y la construcción de otras narrativas acerca del trayecto.

Para abordar el nuevo fenómeno y plantear soluciones, la movilidad urbana es analizada por diferentes autores de las Ciencias Sociales bajo el concepto "Mobility Turn" (Hannam, Sheller, \& Urry, 2006: 1-22), el cual Cresswell define de la siguiente manera:

This turn has been prefigured for a while by a number of theorists across disciplines who have argued for a kind of thinking that takes mobility as the central fact of modern or postmodern life. A significant thrust of these arguments has been to question the perceived prioritization of more rooted and bounded notions of place as the locus of identity (Cresswell, 2010: 551).

Este paradigma incorpora contribuciones desde la ingeniería, la antropología, la sociología, los estudios culturales, la geografía, la sociología, el turismo y las nuevas tecnologías. Una de las primeras corrientes de pensamiento que estudió la movilidad como fenómeno social, fue la Escuela de Chicago (Tapada, 2012: 3), con Robert Park como uno de sus principales exponentes (Sheller \& Urry, 2000), quien concebía la movilidad como una necesidad inherente al ser humano, así como lo es establecerse en un lugar para vivir.

Desde el campo de la antropología uno de los etnógrafos contemporáneos que ha contribuido a la conceptualización del término es James Clifford, quien evita utilizar el término desplazamiento, pues lo considera muy neutral y prefiere llamarlo "viaje", como "término de comparación cultural" que le permite asociarlo con "organismos pertenecientes a un género, y un fenotipo, privilegios de clase, medios de transporte específicos, rutas transitadas, agentes, fronteras, documentos y cosas por el estilo" (Clifford, 1995: 20).

El viaje entonces, sea de proximidad o lejanía, cotidiano o poco frecuente, es un asunto práctico cargado de experiencias complejas y elementales, es una situación personal, inmersa en el flujo colectivo de lo público. El tiempo de la vida que 


\section{perifèria}

Número 19 (2), Diciembre 2014

revistes.uab.cat/periferia

transcurre durante el viaje, hace parte de las experiencias que constituyen la movilidad urbana (Delgado, 1999).

De acuerdo con estas premisas, se podría definir la movilidad como elemento estructurante de los modos de vida urbanos, que se recodifica constantemente como por ejemplo al salir de la casa al lugar de trabajo, para ir de compras, a estudiar, o llevar a los niños al colegio.

Cresswell (2010) plantea dos conceptos implícitamente relacionados con el nuevo paradigma de la movilidad: jerarquía y clase social. Son un conjunto de disposiciones, -parafraseando a Bourdieu (1997)- o prácticas que el individuo realiza en relación con su posición social y que dan origen a un habitus, que lo lleva a tomar una elección y a asumir una posición en sus prácticas cotidianas de movilidad. En este orden de ideas, la jerarquía y la clase social se constituyen en prácticas sociales en el ámbito de la movilidad, de acuerdo con Bourdieu y que además aportan a la construcción de nuevas narrativas acerca del trayecto.

Estas narrativas se constituyen en categorías que tienen origen en los motivos, tiempos, espacios, sensaciones, encuentros e inconvenientes, que configuran la experiencia del viaje y conocer más acerca de ellas puede dar idea de lo que sucede durante el trayecto.

De acuerdo con Cresswell (2010), en el estudio del movimiento de personas y cosas, las metodologías de etnografía móvil y "follow the thing" han desarrollado una serie de herramientas que permiten tener una idea del papel que tiene la movilidad en la producción de jerarquías sociales.

Cresswell propone seis elementos que permiten analizar las narrativas que juegan un rol en la constitución de las jerarquías y las políticas de movilidad y el poder que encierra cada una de ellas en la práctica social.

Estos elementos son los siguientes:

-"Why does a person or thing move? An object has to have a force applied to it before it can move." (Cresswell, 2010:22) (La razón por la cual viajamos: fuerzas internas y externas). 


\section{perifèria}

Número 19 (2), Diciembre 2014

revistes.uab.cat/periferia

- "How fast does a person or thing move? Velocity is a valuable resource and the subject of considerable cultural investment." (Cresswell, 2010:23) (La velocidad del viaje: acceso y exclusividad para ir más rápido, la decisión cultural y política de ir más despacio "the Slow Movement", la cultura de la inmediatez).

-"What rhythm does a person or thing move? Rhythm is an important component of mobility at many different scales." (Cresswell, 2010:23) (EI ritmo de la movilidad: los horarios y los ritmos de vida: corporales, los impuestos por las sensaciones tales como el peligro, y los impuestos por la conectividad y la tecnología).

-"What route does it take? Mobility is channeled. It moves along routes and conduits often provided by conduits in space." (Cresswell, 2010:24) (Las rutas que tomamos para movernos: reparto modal y el privilegio socioeconómico de unos medios sobre otros).

-"How does it feel? Human mobility, like place, surely has the notion of experience at its centre. "The Bob Dylan's question"." (Cresswell, 2010:25) (La experiencia del viaje; cómo nos sentimos durante el viaje).

-"When and how does it stop? Or to put it another way--what kind of friction does the mobility experience?" (Cresswell, 2010:26) (Las fricciones de la movilidad: cuándo, cómo y por qué un individuo se detiene).

Estas preguntas han motivado el estudio de caso que se presenta en los siguientes apartados.

\section{La movilidad en Barcelona}

La estructura del territorio y la manera como está organizada la ciudad, determinan las estrategias de los viajeros para desplazarse, sus actividades cotidianas y el tiempo que invierten en ellas.

La movilidad cotidiana se ve afectada por procesos colectivos como la división del trabajo, la diferenciación de espacios, la estratificación social y la interculturalidad; 


\section{perifèria}

Número 19 (2), Diciembre 2014

revistes.uab.cat/periferia

así como por procesos individuales como el ciclo de vida, el modus vivendi, la estructura familiar y las distintas estrategias que tienen los individuos para acceder a un bien o un servicio o establecer contacto con el otro.

Dentro de la movilidad cotidiana cada vez es más difícil establecer una relación directa entre distribución horaria y cantidad de desplazamientos. Factores como la flexibilidad laboral, las prácticas cotidianas urbanas, la tecnología y la cantidad de actividades que realizan las personas en su tiempo libre, han hecho que en Barcelona las horas punta y las horas valle cada vez sean más difusas, pues los motivos de desplazamiento se multiplican, y los motivos productivos 0 reproductivos tienen la misma relevancia (Miralles-Guasch, 2011; Carme MirallesGuasch \& Freixa, 2008).

De acuerdo con la Enquesta de Mobilitat en Dia Feiner (EMEF) 2012 (IERMB, 2012) en la Región Metropolitana de Barcelona se presentan 16.125.464 desplazamientos en días laborales, que representan 3,8 desplazamientos diarios por persona (ver tabla 1 ). El $40,6 \%$ de los motivos que generan estos viajes son personales y $16 \%$ son por trabajo o estudio. El otro $43,4 \%$, se clasifican como viajes de regreso a casa, de los cuales es importante resaltar que las rutas y el patrón no son similares al viaje de partida de casa y por tal motivo se clasifican distinto. 


\section{perifèria}

Número 19 (2), Diciembre 2014

revistes.uab.cat/periferia

Tabla 1: Distribución de los desplazamientos en días laborables

\begin{tabular}{|l|c|}
\hline \multicolumn{1}{|c|}{ Motivo de desplazamiento } & Porcentaje \\
\hline Trabajo & $12.4 \%$ \\
\hline Estudio & $3,5 \%$ \\
\hline \multicolumn{1}{|c|}{ Total movilidad ocupacional } & $\mathbf{1 6 \%}$ \\
\hline Compras & $10 \%$ \\
\hline Médico/hospital & $2,1 \%$ \\
\hline Visita amigos/familiar & $4 \%$ \\
\hline Acompañar personas & $9,4 \%$ \\
\hline Gestiones personales & $4,8 \%$ \\
\hline Ocio/diversión/comidas/deporte & $6,3 \%$ \\
\hline Paseo & $3,9 \%$ \\
\hline Total movilidad personal & $\mathbf{4 0 , 6 \%}$ \\
\hline Regreso a casa ocupacional & $11,9 \%$ \\
\hline Regreso a casa personal & $31,5 \%$ \\
\hline Total regreso a casa & $\mathbf{4 3 , 4 \%}$ \\
\hline
\end{tabular}

Fuente: EMEF 2012

De estos desplazamientos, el $85,6 \%$ son de conexión interna, es decir, tienen su origen y destino en la ciudad de Barcelona; el 12,8\% entre puntos de la Región Metropolitana de Barcelona (RMB), y 1,5\% por fuera de la RMB. De acuerdo con Miralles-Guasch, "durante los días festivos los desplazamientos por fuera de la RMB se duplican en números absolutos como consecuencia de la realización de más actividades personales" (Miralles-Guash \& Oliver, 2008:122).

Con respecto al medio de transporte utilizado, 52,6\% de los desplazamientos en el interior de Barcelona se hacen en medios no motorizados; $29,8 \%$ en transporte público y $17,6 \%$ en transporte privado. Lo que lleva a concluir que $47,4 \%$ de los viajes se hacen en transporte motorizado y la movilidad en vehículo privado aumenta a medida que crece la distancia por fuera del ámbito de Barcelona ciudad, 


\section{perifèria}

Número 19 (2), Diciembre 2014

revistes.uab.cat/periferia

mientras que los recorridos en transporte público disminuyen.

Otro aspecto importante de estos desplazamientos, es que en los realizados fuera del ámbito de la ciudad de Barcelona, predomina la movilidad ocupacional (33,5\%), mientras que al interior, la mayoría son por movilidad personal $(42,7 \%)$.

La elección de un medio de transporte depende de la subjetividad de cada persona, además del coste del viaje, la distancia a recorrer, la infraestructura y la oferta de medios de transporte, así como la accesibilidad a ciertos lugares.

De acuerdo con Miralles-Guasch, los desplazamientos por motivos personales tienden a realizarse en medios no motorizados, al tener la opción de elegir el destino, mientras que los viajes ocupacionales se hacen en transporte motorizado, a pesar de los inconvenientes y costes que estos puedan generar. Sin embargo existen otras gradaciones relacionadas con las percepciones y las narrativas, vinculadas a subjetividades y a factores culturales, que abordaremos más adelante en este artículo.

Por lo que se refiere a la edad, así como el género, son variables importantes que también influyen en el reparto modal en la RMB (ver tabla 2). De acuerdo con la EMEF2012, las mujeres entre los 30 y 64 años son el grupo poblacional con mayor movilidad, con 4,3 viajes en promedio por día, seguida de los hombres en este mismo rango de edad, con 3,9 viajes. 


\section{perifèria}

Número 19 (2), Diciembre 2014

revistes.uab.cat/periferia

Tabla 2. Movilidad según edad y género por tipo de movilidad

\begin{tabular}{|c|c|c|c|c|c|}
\hline Género & Edad & $\begin{array}{c}\text { Movilidad } \\
\text { ocupacional }\end{array}$ & $\begin{array}{l}\text { Movilidad } \\
\text { personal }\end{array}$ & $\begin{array}{c}\text { Regreso a } \\
\text { casa }\end{array}$ & Total \\
\hline \multirow[t]{4}{*}{ Hombres } & De 16 a 29 años & $26,1 \%$ & $29,0 \%$ & $45,0 \%$ & $100,0 \%$ \\
\hline & De 30 a 64 años & $22,7 \%$ & $34,3 \%$ & $43,1 \%$ & $100,0 \%$ \\
\hline & De 65 y más años & $0,8 \%$ & $53,6 \%$ & $45,7 \%$ & $100,0 \%$ \\
\hline & Total & $19,1 \%$ & $37,0 \%$ & $43,9 \%$ & $100,0 \%$ \\
\hline \multirow[t]{4}{*}{ Mujeres } & De 16 a 29 años & $28,0 \%$ & $26,9 \%$ & $45,1 \%$ & $100,0 \%$ \\
\hline & De 30 a 64 años & $13,7 \%$ & $44,1 \%$ & $42,2 \%$ & $100,0 \%$ \\
\hline & De 65 y más años & $1,1 \%$ & $54,5 \%$ & $44,4 \%$ & $100,0 \%$ \\
\hline & Total & $13,4 \%$ & $43,5 \%$ & $43,0 \%$ & $100,0 \%$ \\
\hline Total & & $16,0 \%$ & $40,6 \%$ & $43,4 \%$ & $100,0 \%$ \\
\hline
\end{tabular}

Fuente: EMEF 2012

Los contrastes altamente significativos se dan entre los motivos y los medios de transporte usados por los diferentes grupos poblacionales. En términos generales, las mujeres de la RMB entre los 16 y 64 años presentan mayores desplazamientos por motivos personales que los hombres, que de acuerdo con Hanson (2010), lo cual obedece a motivos relacionados con actividades como las compras, compañía a amigos o a los hijos. Los hombres adultos por su parte tienen desplazamientos más pendulares, que tienden a ser entre sus casas y los lugares de trabajo (Abril, Romero, \& Borràs, 2009).

Con respecto a los usos de los medios de transporte, se puede observar que las mujeres son las que más se desplazan en medios no motorizados y en medios motorizados de transporte público. Un factor cultural de alta resonancia es que las mujeres entre los 16 y 29 años tienen menor acceso a transporte privado que los hombres en este mismo rango de edad, según lo plantea Miralles-Guash \& Oliver, 2008 (2008: 128). 


\section{perifèria}

Número 19 (2), Diciembre 2014

revistes.uab.cat/periferia

En cuanto a la movilidad, en la Región Metropolitana de Barcelona las personas laboralmente activas que se encuentran en situación de paro son las que presentan mayor número de desplazamientos por día $(4,3)$, seguidamente de los estudiantes $(3,5 \%)$ y los jubilados $(3,3)$ son los grupos que tienen menos viajes durante un día laborable.

En relación con los usos de los medios de transporte, la población activa ocupada es la que más usa el transporte privado para desplazarse (41,5\%), pero también el otro $37,6 \%$ de esta población lo hace a pie o en bicicleta. En este último segmento se manifiesta que la proximidad sigue siendo un factor clave de la movilidad en la RMB.

\section{Datos y método}

Como parte de este estudio de carácter exploratorio, además de la observación participante realizada en la zona geográfica situada en el barrio La Maternitat i San Ramon, de Barcelona, durante los meses de mayo y julio se llevaron a cabo 18 entrevistas en profundidad a sujetos entre los 18 y 64 años de edad, con la ayuda de cuestionarios semiestructurados y grabaciones de audio. Dentro de la selección de casos se incluyeron a funcionarios del Ayuntamiento de Barcelona encargados de ejecutar la política pública de movilidad. Esta información fue complementada con fuentes documentales secundarias relacionadas con la movilidad del barrio y del área metropolitana de Barcelona.

El carácter del estudio aconseja hablar de "selección de casos" más que de "muestras", las cuales implican los conceptos de marco muestral, selección aleatoria y representatividad con un margen de error, implicaciones que en modo alguno nos proponemos plantear.

La selección se ha llevado a cabo a partir de unos criterios que permiten incluir personas que cumplen con determinadas características y excluyen a las que no las tienen (Wimmer \& Dominick, 1996: 69-70). La selección de la muestra la hicimos bajo la técnica de Bola de Nieve, que de acuerdo con Corbetta, 2007:288), "consiste en identificar a los sujetos que se incluirán en la muestra a partir de los propios entrevistados. Se parte de una pequeña cantidad de individuos que 


\section{revista de recerca i formació en antropologia}

\section{perifèria}

Número 19 (2), Diciembre 2014

revistes.uab.cat/periferia

cumplen los requisitos exigidos, y que se utilizan como informadores para localizar a otros individuos con características idénticas".

Los criterios que se definieron para la selección de casos fueron:

- $\quad$ Sujetos afectados por el proyecto de Supermanzana del distrito de Les Corts, ubicado en el barrio La Maternitat i San Ramón.

- $\quad$ Sujetos con un rango de edad entre los 18 y 64 años, de ambos géneros, de variadas situaciones profesionales, con fin de tener un espectro amplio de las diferentes narrativas construidas alrededor de la movilidad.

El ámbito de estudio se ubica entonces entre las siguientes calles, de acuerdo con los datos suministrados por la Agencia de Ecología Urbana de Barcelona:

- $\quad$ Jardines de Bacardí y los Jardines de Josep Goday y Casals.

- Las calles conectoras de la supermanzana: Calle de Regent Mandieta, Felipe de Paz y María Barrients, como continuidad del eje de conexión hacia el barrio de Les Corts.

- $\quad$ Calle de Benavent, como conexión entre la Avenida Madrid y el Camp Nou.

- $\quad$ Calle de Emérita Augusta, como refuerzo de la conexión estratégica desde la Av. Madrid hacia las calles de Felipe de Paz y Benavent. 


\section{perifèria}

Número 19 (2), Diciembre 2014

revistes.uab.cat/periferia

Gráfico 1. Ubicación de paradas del transporte público en Maternitat i San Ramon

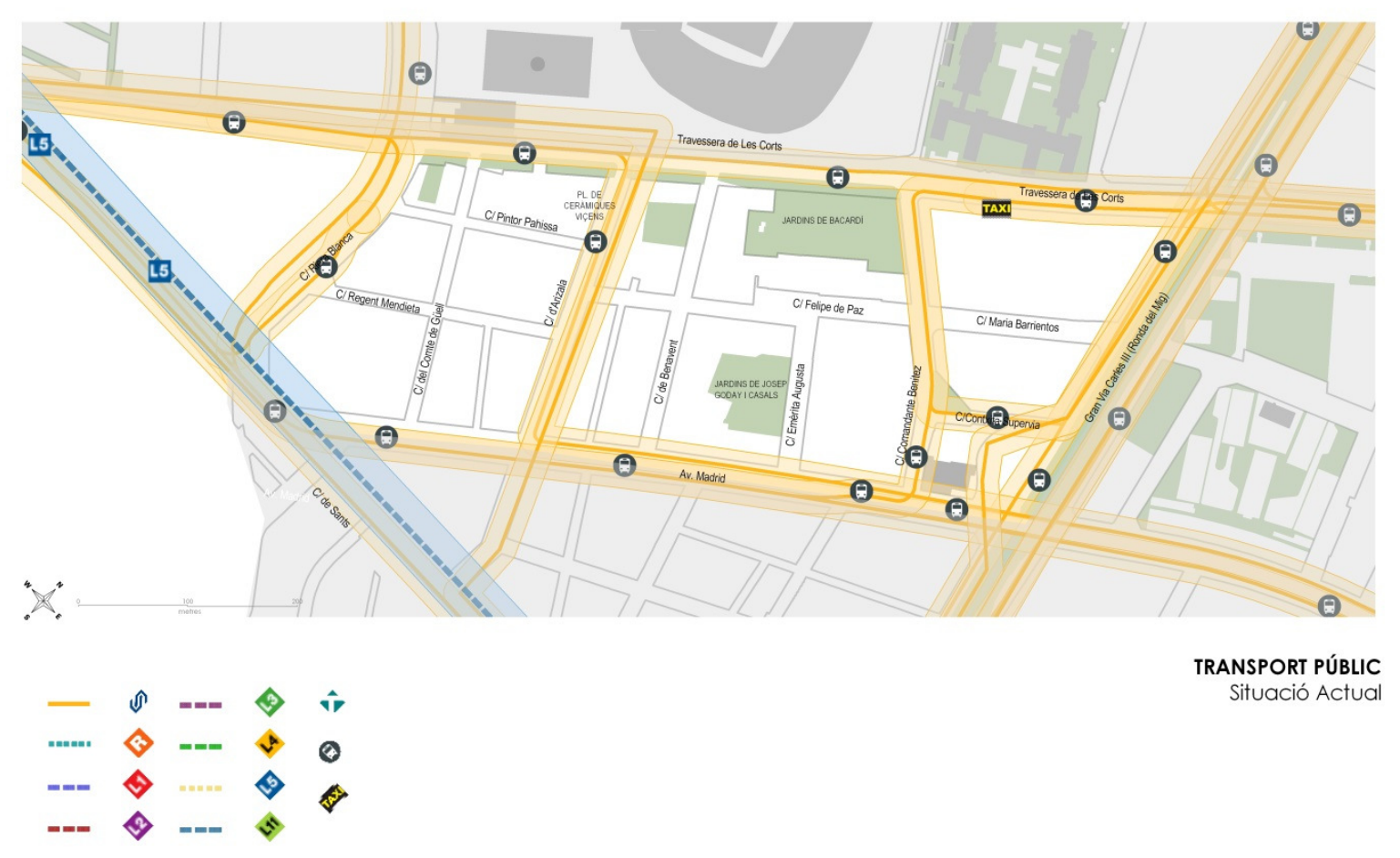

Fuente: Agencia de Ecología Urbana de Barcelona.

Con el análisis de contenido de las entrevistas en profundidad y la información obtenida de la Encuesta de Movilidad del área metropolitana de Barcelona en Días Laborales (EMEF), se estableció una relación con los principios de movilidad expuestos por Cresswell y las jerarquías de movilidad existentes entre los habitantes del sector y las jerarquías de movilidad impuestas por la planificación.

Las unidades se escogieron con base en el "análisis de afirmaciones" (Aigneren, 2009:13) que proporcionan la frecuencia con que ciertos hechos, situaciones o personas se caracterizan de una determinada manera, por ejemplo, la dependencia tácita o la centralidad cultural que tiene el automóvil privado en los entrevistados, 


\section{perifèria}

Número 19 (2), Diciembre 2014

revistes.uab.cat/periferia

es una variable relevante para observar y aproximarse a la comprensión del fenómeno de la movilidad cotidiana en Barcelona.

En este sentido los conceptos de partida fueron: las razones por las cuales una persona se mueve de casa; el tiempo de la movilidad y sus valoraciones; el ritmo de vida y las variables asociadas, tales como los horarios de trabajo, la planeación, las sensaciones durante el viaje; el coste de moverse y los privilegios socioeconómicos para poder elegir una ruta; la utilización de las TIC; el acceso a los medios de transporte, y el uso subjetivo de ellos.

También se partió de las categorías analíticas de Cresswell (2010) para diseñar la entrevista en profundidad y establecer una codificación inicial de la información, la cual se hizo con la ayuda del software Nvivo.

Se crearon 19 codificaciones que dieron lugar a la construcción de seis macroconceptos. Estos conceptos presentan las narrativas que se aproximan a la movilidad cotidiana en un sector determinado de la ciudad de Barcelona.

\section{La movilidad en el barrio La Maternitat i San Ramon: Análisis y resultados}

Para este estudio exploratorio se marcó la tarea de subrayar cuáles son las narrativas culturales que están asociadas con la movilidad cotidiana y cómo estás podrían tener un grado de relación con el reparto modal y con la percepción de la movilidad en Barcelona, tanto a nivel individual como colectivo.

La movilidad está organizada en términos de jerarquías, con unas élites que viajan frecuentemente cortas o largas distancias con pocas fricciones y relativa comodidad. Hay otras clases cuyos desplazamientos son más obligados, tienen más limitantes de tiempo y espacio, por lo que la experiencia está altamente regulada por el acceso a los medios de transporte. Estas premisas como ejes fundamentales de las hipótesis son las que a continuación se contrastan con los hallazgos del trabajo de campo. 


\section{perifèria}

Número 19 (2), Diciembre 2014

revistes.uab.cat/periferia

\section{Viajamos para cumplir nuestra tarea diaria}

La primera hipótesis plantea que la movilidad está subjetivamente organizada en una jerarquía de desplazamientos a nivel individual que van desde lo cotidiano, obligado y rutinario, a lo excepcional, voluntario y valorado.

El trabajo de campo permitió inferir que la movilidad ocupacional tiene un lugar central en la jerarquía de los desplazamientos, hasta tal punto, como se observó en el análisis de los datos, que dentro de las narrativas llega a considerarse como la única movilidad que existe en la vida cotidiana y el resto son secundarias o están inmersas o supeditadas a los tiempos y las distancias.

Entre todos los medios de transporte que fueron punto de discusión con los sujetos, el automóvil fue el medio de transporte, que consciente o inconscientemente fue identificado como un elemento central de sus prácticas cotidianas de movilidad ocupacional. Aunque no fuese de uso frecuente, siempre estuvo y está presente en las narrativas como un factor de comparación con otros medios: como un elemento aspiracional -en el caso de los más jóvenes-, o como una herramienta de trabajo con alto grado de dependencia para realizar la labor.

También se observó que la falta de espacios de aparcamiento es un elemento común entre los propietarios de automóviles, que demandan más zonas de aparcamiento, argumentando que se trata de un derecho. La gestión del aparcamiento es un elemento de disuasión y control por parte de las autoridades para regular el número de vehículos rodando por la ciudad.

De acuerdo con los planteamientos teóricos, el automóvil es un medio de transporte tradicionalmente asociado a la velocidad, a la independencia y a la sensación de libertad (Featherstone et al., 2005). Hoy el valor más apreciado por los propietarios entrevistados, es la comodidad, palabra que encierra variables de precio, seguridad, funcionalidad y confort. 


\section{revista de recerca i formació en antropologia}

\section{perifèria}

Número 19 (2), Diciembre 2014

\section{revistes.uab.cat/periferia}

El espacio para aparcar y la comodidad son dos variables que resultaron contrapuestas durante el análisis. Son variables interdependientes que en las narrativas de la movilidad a mediano plazo podrían destronar al automóvil de su lugar central en la jerarquía de la movilidad de Barcelona.

El espacio para aparcar ocupa entre los sujetos un lugar central e incluso más importante que el precio del combustible, como lo planteó uno de ellos: "éste ya es un costo que se tiene asumido."

Se halló entonces que el binomio automóvil-parking es una variable que en las narrativas de la movilidad está presente en lo cotidiano, obligado y rutinario e incluso hasta en los desplazamientos excepcionales, voluntarios y que son altamente valorados. Según Sheller y Urry (2000:739), son seis los componentes entrelazados que determinan el alto grado de dominación del automóvil en la sociedad. En este estudio aproximativo, el componente de espacio (Environmental resource-use) resultó ser central en las narrativas de movilidad cotidiana, por encima de "manufactured object", "individual consumption", "machinic complex", "'quasi-private' mobility" o "dominant culture."

\section{El precio de la comodidad}

Otra de las hipótesis plantea que las jerarquías de valoración de la movilidad están relacionadas con el nivel socioeconómico de las personas. En este punto confluyen variables como la organización del territorio y la de las ciudades, que de acuerdo con Miralles-Guasch \& Freixa (2008:9), determinan la manera como las personas hacen uso del tiempo y distribuyen sus ingresos para moverse por la ciudad de manera eficiente.

A lo largo de la investigación constamos con que el coste económico (considerado costoso por todos los sujetos) de moverse en un medio de transporte, es un valor que siempre es tenido en cuenta a la hora de elegir un medio de transporte Sin embargo, no es un factor determinante. La racionalidad económica se evalúa en conjunto con la funcionalidad, la seguridad y el confort, valores que constituyen la representación de la comodidad. 


\section{perifèria}

Número 19 (2), Diciembre 2014

revistes.uab.cat/periferia

Se debe tener en cuenta que los sujetos escogidos para la muestra, poseían una condición socioeconómica desahogada, por lo que fue común encontrar que la comodidad y el precio tenían una importancia similar. El destino es el que determina cuál es el medio más idóneo para llegar. Se observó que muchos de los sujetos usan el automóvil debido a que resulta más barato y cómodo para acceder a determinados destinos que el transporte público no cubre.

La búsqueda de la comodidad de la que siempre hablaron los sujetos entrevistados hace pensar que los viajes, tal como lo planteaba Giglia (2012) y Clifford (1995), sean de proximidad o lejanía, cotidianos o poco frecuentes, son un asunto práctico cargado de experiencias complejas y elementales, en donde la comunicación y la cultura juegan roles determinantes. Fue a partir de experiencias creadas de percepciones propias o escuchadas, que los sujetos entrevistados valoraron temas como la seguridad, o el ir en bicicleta, pero también apelaron a situaciones básicas como las experiencias sensoriales relacionadas con cada medio, para decidir cuál era mejor que otro, independientemente de la velocidad que este pudiera ofrecer.

\section{El nuevo valor de la proximidad}

La existencia de una organización urbana monofuncional heredada de la modernidad, está llamada a cambiar, lo que obligará a las ciudades a crear nuevas centralidades o a regresar al centro (Urry, 2002).

El valor de la proximidad fue calificado por el grupo de sujetos entrevistados de forma positiva. Sin embargo es aún prematuro considerar este aspecto como eje central de la jerarquía de la movilidad cotidiana. Son los sujetos ubicados en el rango de edad joven, los que manifiestan la preocupación y la conveniencia por estar cerca socialmente. No quieren depender ni invertir parte de sus ingresos en medios de transporte. Hace doce años Urry planteó que la tendencia era que

"prosperous young single people to prefer not suburban but city-centre living focused around socialities within public spaces of bars, leisure clubs, restaurants and nightclubs. This gentrification involves a lack of distance between work and home so as to sustain the co-presence of 'tight social 


\section{perifèria}

Número 19 (2), Diciembre 2014

revistes.uab.cat/periferia

worlds' within city centres" (2002: 261).

El alcance de este estudio no permite comprobar dicha hipótesis, sin embargo fue claro que dentro de la muestra de sujetos con edades comprendidas entre 18 y 29 años, la cercanía fue un factor valorado y relacionado con la movilidad personal.

\section{Conclusiones}

La movilidad ocupacional tiene un lugar central en la jerarquía de los desplazamientos. Es importante considerar con base en los testimonios recogidos, en la experiencia de campo y en el análisis bibliográfico, que los desplazamientos obligados por razones de trabajo o estudio, no pueden analizarse únicamente desde la racionalidad económica.

Los sujetos de la muestra, además del coste económico, manifiestan otras variables a la hora de realizar el viaje, tales como el destino, los eventos que pueden resultar durante el desplazamiento y otras motivaciones personales. Esta combinación de variables culturales determina la elección de un medio de transporte y podría estar afectando el reparto modal de la ciudad.

De los datos obtenidos se puede deducir que la movilidad obligada es hegemónica, su relación con la movilidad personal no puede analizarse de manera vertical, sino que obedece a un orden complejo característico de la sociedad líquida (Bauman, 2005), concepto teórico que fue clave en la estructuración de este estudio con fines aproximativos.

La valoración que se hace de la movilidad en Barcelona, así como sus narrativas, están directamente relacionadas con los transportes motorizados, por lo que se concluye que los desplazamientos a pie, aunque son valorados, no se les da la importancia y el reconocimiento que tienen en relación con la ventaja que representa hoy la proximidad. Es un punto que debe estudiarse con mayor profundidad.

La comodidad fue el atributo más valorado por el grueso de la muestra, pero esta no debe analizarse únicamente en relación con la definición del concepto de 


\section{perifèria}

Número 19 (2), Diciembre 2014

revistes.uab.cat/periferia

"conveniente, oportuno, acomodado, fácil y proporcionado."

La comodidad de acuerdo con las observación realizadas en el trabajo de campo, está directamente vinculada con las experiencias del viaje y los imaginarios que hay alrededor del lugar de tránsito, en el que operan variables subjetivas que causan "agobio" en el viajero, pero también están presentes en las narrativas de viaje, los significados simbólicos, la fragmentación y la diversificación social que plantea Giglia (2012:60) y que contribuyen a la construcción del imaginario de la comodidad, el cual compite o está directamente relacionado con el coste económico y el tiempo. Se requiere de un estudio de mayor profundidad para establecer cuál es la relación.

Aunque el uso de las tecnologías de la información y la comunicación no era el objetivo de análisis de este trabajo, se infirió que estuvo presente en las narrativas asociadas a la ruta y forma parte de los factores que constituyen el atributo de la comodidad. La tecnología tiene diversos usos antes y durante el recorrido, su utilización es generalizada entre los viajeros, con mayor proporción en el grupo de los jóvenes, que consultan con frecuencia acerca del recorrido y las variables asociadas. Fue evidente su importancia, especialmente el uso de plataformas tecnológicas para resolver dudas relacionadas con la superación de obstáculos, atascos, cierre de vías, nuevas rutas y otros eventos

En definitiva, esta breve incursión en el campo de la movilidad y sus narrativas nos permite identificar algunos elementos de interés pero también nos abre nuevos y estimulantes horizontes.

\section{Bibliografía}

Abril, P., Romero, A., \& Borràs, V. (2009). Los hombres y sus tiempos: hegemonía, negociación y resistencia. Barcelona, p.84.

Aigneren, M. (2009). Análisis de contenido. Una introducción. La sociología en sus escenarios, 1-52.

Banister, D. (2011). Cities, mobility and climate change. Journal of Transport Geography, 19(6), 1538-1546. doi:10.1016/j.jtrangeo.2011.03.009 


\section{perifèria}

Número 19 (2), Diciembre 2014

revistes.uab.cat/periferia

Bauman, Z. (2005). Amor líquido: acerca de la fragilidad de los vínculos humanos. Madrid [etc.]: Fondo de Cultura Económica.

Bourdieu, P. (1997). Razones prácticas.

(1994). Sobre la teoría de la acción (p. 225). Barcelona: Anagrama.

Clifford, James. (1995). Las culturas del viaje. Revista de Occidente, (170), 1-21.

Corbetta, P. (2007). Metodología y técnicas de investigación social. Madrid: McGraw-Hill.

Cresswell, T. (2006). On the move: mobility in the modern Western world. New York: Routledge.

Cresswell, T. (2010). Mobilities I: Catching up. Progress in Human Geography, 35(4), 550-558. doi:10.1177/0309132510383348

Delgado, M. (1999). El Animal público: hacia una antropología de los espacios urbanos. Barcelona: Anagrama.

Featherstone, M., Thrift, N., \& Urry, J. (2005). Automobilities. Londres: SAGE.

Giglia, A. (2012). El Habitar y la cultura: perspectivas teóricas y de investigación. México: Universidad Autónoma Metropolitana, Unidad Iztapalapa, División de Ciencias Sociales y Humanidades.

Gutiérrez, J., \& García-Palomares, J. C. (2007). New spatial patterns of mobility within the metropolitan area of Madrid: Towards more complex and dispersed flow networks. Journal of Transport Geography, 15(1), 18-30. doi:10.1016/j.jtrangeo.2006.01.002

Hagman, O. (2003). Mobilizing meanings of mobility: car users' constructions of the goods and bads of car use. Transportation Research Part D: Transport and Environment, 8(1), 1-9. doi:10.1016/S1361-9209(02)00014-7

Hannam, K., Sheller, M., \& Urry, J. (2006). Editorial: Mobilities, Immobilities and Moorings. Mobilities, 1(1), 1-22. doi:10.1080/17450100500489189

Hanson, S. (2010). Gender and mobility: new approaches for informing 


\section{perifèria}

Número 19 (2), Diciembre 2014

revistes.uab.cat/periferia

sustainability. Gender, Place \& Culture, 17(1), 5-23. doi:10.1080/09663690903498225

IERMB. (2012). Enquesta de mobilitat en dia feiner 2012 (resultados). Barcelona, p.8.

Jacobs, J. (2013). Muerte y vida de las grandes ciudades ( $3^{\circ}$ ed., p. 488). Madrid: Capitán Swing Libros S.I.

Kaufmann, V. (2002). Re-Thinking Mobility: Contemporary Sociology (p. 112). Hampshire: Ashgate Publishing Company.

Kenworthy, J. R., \& Laube, F. B. (1999). Patterns of automobile dependence in cities: an international overview of key physical and economic dimensions with some implications for urban policy. Transportation Research Part A: Policy and Practice, 33(7-8), 691-723. doi:10.1016/S0965-8564(99)00006-3

Klinger, T., Kenworthy, J. R., \& Lanzendorf, M. (2013). Dimensions of urban mobility cultures - a comparison of German cities. Journal of Transport Geography, 31, 18-29. doi:10.1016/j.jtrangeo.2013.05.002

Miralles-Guasch, C. (2011). Dinámicas metropolitanas y tiempos de la movilidad. La región metropolitana de Barcelona, como ejemplo. Anales de Geografía de La Universidad, 31, 125-145. doi:10.5209/rev

Miralles-Guasch, C., \& Cebollada, À. (2009). Movilidad cotidiana y sostenibilidad, una interpretación desde la geografía humana. Boletín de La AGEN o, 193-216.

Miralles-Guasch, C., \& Freixa, E. (2008). L'Ús social del temps a la regió metropolitana de Barcelona. Barcelona: Pla Estratègic Metropolità de Barcelona.

Miralles-Guash, C., \& Oliver, L. (2008). La Mobilitat quotidiana a Catalunya. Retrieved from http://cataleg.uab.cat/record=b1763687 S1*cat

Passalacqua, A. Batteau, A. (2014). Imagined mobilities. Mobile Lives Forum.

Sennett, R. (1997). Carne y piedra: el cuerpo y la ciudad en la civilización occidental. Madrid: Alianza.

Sheller, M., \& Urry, J. (2000). The city and the car. International Journal of Urban 


\section{perifèria}

Número 19 (2), Diciembre 2014

revistes.uab.cat/periferia

and Regional, 24(December 2000).

Sheller, M., \& Urry, J. (2006). The new mobilities paradigm. Environment and Planning A, 38(2), 207-226. doi:10.1068/a37268

Tapada, M. T. (2012). Sobre el concepto de antropología urbana y de antropología del espacio. Documento No Publicado, 1-29.

Urry, J. (2002). Mobility and Proximity. Sociology, 36(2), 255-274. doi: $10.1177 / 0038038502036002002$

Wimmer, R. D., \& Dominick, J. R. (1996). La Investigación científica de los medios de comunicación: una introducción a sus métodos. Barcelona: Bosch. 